\title{
Linearities, Non-Linearities and Phase Transitions in Loss Diffusion Processes in Financial Networks
}

\author{
M. Eboli* \\ Dipartimento di Economia Aziendale, Università 'G. d'Annunzio', Pescara, ITALY
}

(Received 1 December, 2019)

\begin{abstract}
Banks and financial institutions are linked by financial obligations that form complex networks. These networks serve risk sharing purposes and become channels of contagion in the event of liquidity and insolvency shocks. The consequent processes of loss diffusion are usually non-linear and, in some cases, exhibit phase transitions from situations in which there is no default contagion to systemic crisis that involve the entire network. In this paper, I discuss the results of recent numerical simulations of contagion processes in financial networks that present some unexpected linearities.
\end{abstract}

PACS numbers: $89.65 . \mathrm{Gh}$

Keywords: systemic risk, financial contagion, financial networks

DOI: https://doi.org/10.33581/1561-4085-2020-23-2-207-211

\section{Introduction}

In these pages, I discuss the existence of linearities and phase transitions in the diffusion of losses and defaults in networks of financial operators. Banking and financial sectors are characterised by networks of obligations that stem from trading activities, from payment systems and from the cross-holding of short term liabilities that enable banks to share the risk of facing liquidity shortages. Formally, a financial network is represented as a weighted and directed graph, in which the nodes are financial operators (banks and other intermediaries), and the links are the financial obligations that connect pairs of such operators. These networks of financial obligations become the vehicles of financial contagion in the event of liquidity and insolvency shocks. The liquidity shortage of one bank is transmitted to other banks through the retrieval of shortterm lending, while the default of a bank can induce the default of its creditors if the losses transmitted by the defaulting bank are sufficiently large. Since the financial crisis erupted in 2007, a large and growing body of literature investigates the properties of financial networks, in particular, the dynamics of the contagion processes that can take place in such networks. The study of contagion and systemic risk in financial networks started with the seminal works [1] and [2]( see also

\footnotetext{
*E-mail: m.eboli@unich.it
}

[3] for a review of the subsequent literature.

The withdrawal of funds, due to a liquidity shock, and the transmission of losses, due to an insolvency shock, are both linear in the value of the interbank exposures that form the network, i.e. they are linear in the weights of the links of the network. In other words, the contagion function defined in a financial network at the node level is linear. Nonetheless, in the existing literature on financial contagion, no results indicate a linear relation, at the network level, between the size of an exogenous shock and the scope of the default contagion that it generates. In these pages, I discuss the emergence of unexpected linearities in the contagion processes that take place in regular networks-i.e. in networks in which all nodes have the same indegree and outdegree.

The paper is organised as follows. In the next section, I briefly introduce a model of a financial network and the contagion function defined over it. In section 3, I present some existing analytic results and discuss some numerical results that indicate the existence of unexpected linearities in the contagion functions computed on regular networks endowed with different connectivity. Conclusions are drawn in section 4 .

\section{A financial network and its contagion process}

The model of choice to represent a financial network is a connected, directed and weighted 
graph $N:=(\Omega, \Lambda)$, where the nodes $\omega_{i} \in \Omega$,for $i=1,2, \ldots, n$, represent banks or other financial intermediaries, and the links in $\Lambda \subseteq \Omega^{2}$ represent the financial claims that connect the members of $\Omega$ to one another. The nodes are characterised by the balance sheet (the budget identity) of the corresponding operator $\omega_{i}: a_{i}+c_{i}=h_{i}+d_{i}+$ $e_{i}$, where: i) $a_{i}$, are external assets, i.e. claims on agents that do not belong to $\Omega$, ii) $c_{i}$ are intra-network assets, i.e. claims on other agents in $\Omega$; iii) $h_{i}$ are external debts, i.e. liabilities towards agents that do not belong to $\Omega$; iv) $d_{i}$ are intra-network debts, i.e. liabilities towards other agents in $\Omega$; and, finally, v) $e_{i}$ is the net worth (equity) of the agent. In what follows, I discuss the linearity of contagion processes started by exogenous insolvency shocks, that is, shocks that are defined as a loss of value of the external assets $a_{i}$. Formally, a shock is a vector of scalars $\left[\delta_{i} a_{i}\right], i \in \Omega$, where $\delta_{i}(i=1,2, \ldots, n) \in[0,1]$, at least one $\delta_{i}>0$, and its magnitude is $\sigma=\sum_{i} \delta_{i} a_{i}$.

The diffusion of an insolvency shock across $N$ is governed by the rules of limited liability, debt priority and pro-rata reimbursement of creditors. The limited liability of shareholders is represented by an absorption function

$$
\beta_{i}\left(\lambda_{i}\right)=\min \left(\frac{\lambda_{i}}{e_{i}}, 1\right)
$$

where $\lambda_{i}$ is the loss experienced by a bank $\omega_{i}$, received exogenously or from other banks in $\Omega$. This loss is absorbed by the equity of the bank, $e_{i}$, if $e_{i}-\lambda>0$. Conversely, if $\lambda-e_{i}>0$, then the overflowing loss $\lambda-e_{i}$ is transmitted to the creditors of $\omega_{i}$ in a pro-rata fashion. Let

$$
b_{i}\left(\lambda_{i}\right)=\max \left(0, \frac{\lambda_{i}-e_{i}}{h_{i}+d_{i}}\right)
$$

be the loss-given-default function of a bank in $\Omega$. The variable $b_{i} \in[0,1]$ measures the fraction of the $i$-th bank's debt that is not recovered through bankruptcy procedures. If $b>0$, the bank is insolvent, its assets are liquidated, and its creditors get a pro-rata refund. In this case, a bank $\omega_{j} \in N$ which is a creditor of bank $\omega_{i}$ receives from the latter a loss equal to $b_{i} d_{i j}$, where $d_{i j}$ is the debt of bank $i$ towards bank $j$. The loss $\lambda_{i}$ born by a bank in $\Omega$ is the sum of the losses, if any, received through its external and internal exposures:

$$
\lambda_{i}=\delta_{i} a_{i}+\sum_{j} b_{j} d_{j i}
$$

Given an exogenous shock that hits some banks in $N$, the above-defined absorption and lossgiven-default functions assign a positive amount of losses to each link in $\Lambda$. In so doing, these functions determine the contagion process that takes place in $N$. Formally, given a shock to a network $N$, the consequent distribution of losses is a map $f:\left(L^{\Omega}, L^{A}, L^{T}, L^{H}\right) \rightarrow \mathbb{R}^{+}$such that: $f\left(l_{i}^{k}\right)=b^{k} a_{i}^{k}, f\left(l_{i j}\right)=b_{i} d_{i j}, f\left(l_{H}^{i}\right)=b_{i} h_{i}$, $f\left(l_{T}^{i}\right)=\beta_{i} e_{i}$. This map defines a contagion function that maps the set of possible shocks onto $\Omega$, determining the cardinality of the set of secondary defaults, ${ }^{1}$ i.e. of the set of the banks $\bar{\Omega} \subset \Omega$ that default because of the (endogenous) losses received from their defaulting debtors in $\Omega: \bar{\Omega}:=\left\{\omega_{i} \mid \sum_{j} b_{j} d_{j i}>e_{i}\right\}$. Let $|\bar{\Omega}|=f(\sigma)$ be this contagion function. Given a shock $\sigma^{*}$, there is contagion if $f\left(\sigma^{*}\right)>0$, i.e. if $\bar{\Omega}$ is not empty. For any given set of secondary defaults, there is a smallest shock capable of generating it. We call this shock the threshold of contagion. The first contagion threshold of a network, for instance, is the smallest shock that causes at least one secondary default. The final contagion threshold is the smallest shock that causes the default of all banks in the network.

\section{Linearities, non-linearities and phase transitions in sparse and dense regular networks}

As shown above, the transmission of losses at the node level, i.e. from a defaulting bank to its creditors, is a linear function of the liabilities of a bank. At the network level, however, this linearity of loss transmission does not generate a linear progression of default contagion in generic financial networks. The topology of a network determines the passing of losses along the paths and cycles of the network and the

\footnotetext{
${ }^{1}$ As opposed to the set of primary defaults, i.e. the banks that default because of the exogenous loss of value of their assets $a_{i}$, i.e. the banks for which $\delta_{i} a_{i}-e_{i}>0$
} 
scope of the consequent contagion process. In generic networks, this process leads to uneven, and often complex, allocations of losses among the descendants of the defaulting nodes. As a consequence, the linearity of loss transmission at node level does not imply any linearity in the dynamics of contagion. Interestingly, recent numerical simulations, put forward by Eboli et al. (2019), show that this general result does not entirely apply to the class of regular networks.

Within the class of regular networks, we have analytic results that pin down the response to shocks of complete and ring networks [4,5], which are defined as follows. In a complete interbank network, each bank places a deposit in every other bank. Let $N^{c}:=\left(\Omega, \Lambda^{c}\right)$, where $\Lambda^{c}=$ $\left\{l_{i j} \mid i \neq j ; i, j=1, \ldots, n\right\}$, be a complete interbank network. The latter is the densest network in the class of regular networks, i. e. the networks in which every node has the same indegree and outdegree. In other words, the complete networks are maximally connected and minimally centralised (all nodes have the same centrality). Within the same class of regular networks, the least connected network is the ring network, also known as wheel or unilateral circle. In a ring network, each bank is linked to just one creditor and one debtor bank, forming a chain of obligations that creates a closed path in which the start node and the final node coincide. Formally, a ring network $N^{o}=\left\{\Omega, \Lambda^{o}\right\}$ is such that $L^{\Omega}=$ $\left\{l_{i j} \mid i=1,2, \ldots, n ; j=i+1\right.$ for $i=1, \ldots, n-$ 1 , and $j=1$ for $i=n\}$.

Recent analytic results, put forward in [6] and [5] demonstrate that the response to exogenous shocks of complete networks shows a phase transition. A network $N^{c}$ has a single threshold of contagion, i.e. the smallest shock that can induce one secondary default is also sufficient to induce the collapse of the whole banking system. In other words, in a complete network $N^{c}$ the first and the final thresholds of contagion coincide and are equal to (see [5], proposition 4.)

$$
\tau^{c}=n e_{i}+e_{i} \frac{h_{i}}{d_{i j}} .
$$

In correspondence of this threshold, the contagion function jumps from zero (no secondary defaults) to the largest possible number of secondary defaults, i.e. the cardinality of the set $\left|\Omega \backslash \Omega^{+}\right|$, where $\Omega^{+}:=\left\{\omega_{i} \mid \delta_{i} a_{i}>e_{i}\right\}$ is the set of primary defaults. For this reason, complete networks are known to be robust-yet-fragile to shocks. They are entirely resilient to shocks smaller than $\tau^{c}$ while being completely fragile (systemic crisis) to shocks larger than $\tau^{c}$.

Conversely, the ring networks show the opposite response to shocks: they are vulnerableyet-resilient. Ring networks are vulnerable to episodes of contagion of limited scope when hit by small shocks, while being highly resilient to large shocks. This feature is revealed by the fact that, in ring networks, there is a large gap between the first and last contagion thresholds.

Between the ring network, endowed with $n$ links, and the complete networks, endowed with $n(n-1)$ links, there is the entire range of regular networks with $n \cdot x$ links, for $x=2,3, \ldots, n-2$. For these 'intermediate' regular networks, there are no analytic results about their exposure to default contagion. In a recent work [7] study the response to shocks of regular networks endowed with different degrees of connectivity ${ }^{2}$. In that paper, we run numerical simulations of contagion processes on randomly generated regular networks. We generate networks composed of 64 banks, with different density of connections. We start from ring networks, with $n$ links connecting $n$ nodes, and progressively add links to them generating, in so doing, regular networks of increasing density. In the last step of this re-wiring process, the 63rd step, we obtain the densest regular networks, i.e. the complete network, with $n(n-1)$ links. We then perturb these networks with sequences of random shocks of progressively increasing magnitude. We run 1000 rounds of simulated contagion processes for each pair (network configuration, shock size). In each of these rounds, our simulation device randomly selects a set of primary defaults to shock the system, and progressively augments the cardinality of this set in the following steps of the computation. The simulation device computes the above-defined contagion function for each network configuration and for each shock, recording the corresponding secondary defaults, i.e. the scope of contagion.

\footnotetext{
${ }^{2}$ In the same paper, these authors also study the response to shocks of networks characterised by different degrees of centralisation.
} 
(a)

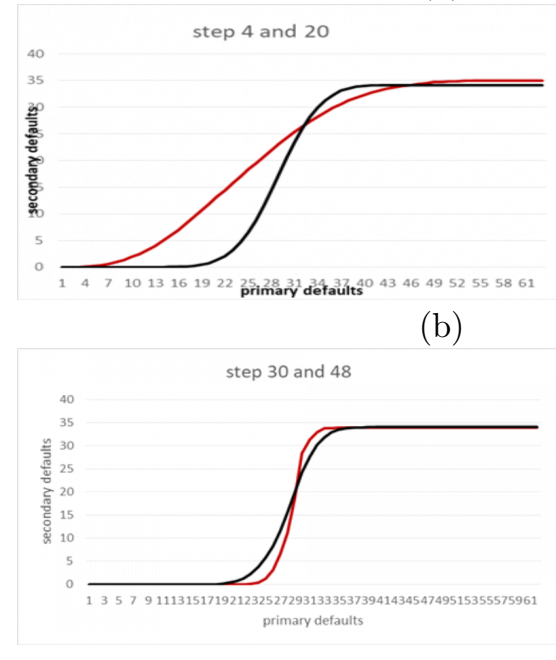

FIG. 1. (color online) Number of secondary defaults as a function of the primary defaults. (a) networks with $n \cdot 4$ and $n \cdot 20$ links, (b) networks with $n \cdot 30$ and $n \cdot 48$ links

Interestingly, these simulations have revealed some unexpected linearities in the dynamics of default contagion in our regular networks.

For the first network configuration, the ring networks, we obtain an increasing and concave contagion function. Starting from the second step of the re-wiring process, i.e. circular networks with $2 n$ links, we obtain S-shaped contagion functions. Surprisingly, we observe that these S-shaped curves are linear in remarkably large neighbourhoods of their inflexion points, as shown in the Figure 1 that plots the number of secondary defaults (scope of contagion) as a function of the primary defaults (exogenous shock). Fig. 1a depicts the results obtained at the fourth and twentieth step of re-wiring, i.e. networks with $n \cdot 4$ and $n \cdot 20$ links, respectively, Fig. 1b depicts the same contagion function obtained with networks with $n \cdot 30$ and $n \cdot 48$ links. This means that, for a medium range of shock magnitudes, the contagion function is well approximated by a linear function $|\bar{\Omega}|=\alpha(\sigma)$, where the scalar $\alpha$ grows in the connectivity of the networks at hand. The fact that these curves become steeper as the density of the networks grow is explained as follows. As connectivity increases, from step 1 to step 63 of the re-wiring process, so does the outdegree of defaulting nodes, i.e. the number of creditors of each defaulting bank in $\Omega^{+}$. Thus,

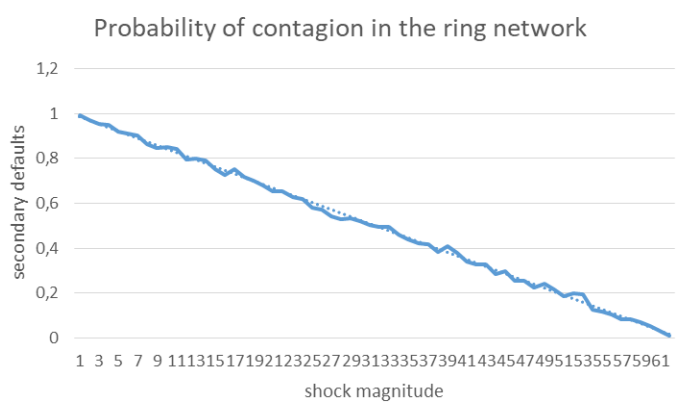

FIG. 2. (color online) Average occurrence of a secondary default for each unit increase of the shock magnitude

the more connected a regular network is, the more the losses diffused across the network by the primary defaults in $\Omega^{+}$are evenly allocated among the banks in $\Omega \backslash \Omega^{+}$. As a consequence, the more connected a regular network is, the more it becomes robust-yet-fragile to shocks and the faster the growth of contagion when shocks approach the final threshold.

Thus, it is not surprising that the scalar $\alpha$ grows in the connectivity of the networks. What is surprising is that-in all the network configurations at hand but the ring and the complete networks, and for a non negligible range of values of the exogenous shock-a unit increase of the shock magnitude generates a constant number $\alpha$ of additional secondary defaults. We do not have an explanation for this striking regularity.

Another unexpected linearity that arises from this set of numerical simulations is the following. We found that, in ring networks, the probability that one unit increase in the number of primary defaults causes one more secondary default is decreasing in an almost linear fashion. Figure 2 shows the average occurrence of a secondary default for each unit increase of the shock magnitude (number of primary defaults) obtained in the 1000 rounds of our simulations on the ring networks. Normalising to unity, we have an estimate of the probability mentioned above. As it appears from the above plot, the discrepancies between the numerical results and a straight line are minimal and can be explained as errors of the statistical approximation. This numerical result strongly suggests that the probability at hand is a decreasing and linear function of the number of primary defaults. 
What makes this finding interesting is the lack of analytic results on this specific issue. The diffusion of defaults in a ring network is closely related to the discrete circle covering problem (see [2] and the literature cited therein). Paper [2] provides the characterisation of some moments of the probability distribution of 'covered' nodes in ring networks and apply them to the analysis of contagion in interbank networks. As the authors put it, "the number of insolvent banks induced by the $k$-th bad bank [primary default, e.n.], becomes a random variable that depends on the location of the other bad banks"([2], page 23). To the best of our knowledge, neither these authors nor others have provided a characterisation of the probability that one more 'bad bank' induces one more bankruptcy.

\section{Conclusions}

The scope of the episodes of default contagion that can occur in financial networks certainly depends on the size of the shocks that cause them. The existing literature presents analytical and computational results that investigate the dynamics of default contagion. None of these results hints at a linear relation between the magnitude of a shock and the extent of the consequent contagion. In these pages, I present some unexpected linearities emerged in numerical simulations of default contagion run over ring networks and regular networks endowed with different degrees of connectivity. These surprising regularities call for an explanation and a formal analysis capable of providing a formal characterisation of the determinants of the linearities at hand.

\section{References}

[1] F. Allen, D. Gale. Financial Contagion. J. Political Economy. 108(1). 1-34 (2000).

[2] J.-C. Rochet, J. Tirole. Interbank Lending and Systemic Risk. Journal of Money, Credit and Banking. 28, 733 (1996).

[3] A. Cabrales, P. Gottardi, F. Vega-Redondo. Financial Contagion in Networks. In: The Oxford Handbook of the Economics of Networks. Ch. 20. (OUP, 2016).

[4] M. Eboli et al. The effects of connectivity and centralization of financial networks on their exposure to default contagion. (working paper) (2019).

[5] F. Castiglionesi and M. Eboli. Liquidity Flows in Interbank Networks. Review of Finance, European Finance Association. 22(4), 1291-1334 (2018).

[6] M. Eboli. A flow network analysis of direct balance-sheet contagion in financial networks. Journal of Economic Dynamics and Control. 103, 205-233 (2019).

[7] G. Barlevy, H. N. Nagaraja. "Properties of the Vacancy Statistic in the Discrete Circle Covering Problem". In: P. Choudhary, C. Nagaraja, H. Ng (eds) Ordered Data Analysis, Modeling and Health Research Methods. Springer Proceedings in Mathematics \& Statistics, vol 149. Springer (2015). 